\title{
ON GROUPS AND COUNTER AUTOMATA
}

\author{
MURRAY ELDER \\ Department of Mathematical Sciences, Stevens Institute of Technology, \\ Hoboken, New Jersey 07030, USA. \\ melder@stevens.edu
}

MARK KAMBITES

School of Mathematics, University of Manchester, Manchester M60 1QD, England.

Mark.Kambites@manchester.ac .uk

GRETCHEN OSTHEIMER

Department of Computer Science, Hofstra University, Hempstead, New York 11549, USA.

cscgzo@hof stra.edu

\begin{abstract}
We study finitely generated groups whose word problems are accepted by counter automata. We show that a group has word problem accepted by a blind $n$-counter automaton in the sense of Greibach if and only if it is virtually free abelian of rank $n$; this result, which answers a question of Gilman, is in a very precise sense an abelian analogue of the Muller-Schupp theorem. More generally, if $G$ is a virtually abelian group then every group with word problem recognised by a $G$ automaton is virtually abelian with growth class bounded above by the growth class of $G$. We consider also other types of counter automata.
\end{abstract}

\section{INTRODUCTION}

Blind counter automata, and the languages they accept, were introduced and extensively studied by Greibach [14, 15]. Such an automaton is a finite state acceptor augmented with a number of integer counters; these are all initialised to zero, and can be incremented and decremented during operation, but not read. The automaton accepts a word exactly if it the reaches an accepting state, with the counters all returned to zero.

At the same time, an area of considerable interest in combinatorial group theory is the study of word problems of finitely generated groups; it is now well-known that many structural properties of groups correspond naturally to language theoretic properties of their word problems, and vice versa [1, 19, 20, 26. One of the main objectives of this paper is to give a complete characterisation of the class of groups whose word problems are blind counter languages, thus answering a question posed by Gilman [11. 
Theorem 1. Let $H$ be a finitely generated group. Then the word problem for $H$ is accepted by a blind $n$-counter automaton if and only if $H$ is virtually free abelian of rank $n$ or less.

As well as being of interest in its own right, Theorem 1 forms an important part of a more general framework. Blind counter automata can be viewed as a class of $G$-automata or blind group automata. Recall that a $G$-automaton is a finite state acceptor augmented with a memory register which stores an element of a given monoid or group; the automaton cannot read the content of its register, but it may change the value by multiplying on the right by some element of the monoid. The register is initialised with the identity element, and the automaton accepts an input word $w$ exactly if, by reading this word, it can reach a final state with the register returned to the identity. Many important classes of languages can be characterised as $G$-automaton languages for particular groups $G$; these include the context-free languages [3, 5, 22] and the recursively enumerable languages [25]. It follows easily from the definitions that blind $n$-counter automata are the same thing as $\mathbb{Z}^{n}$-automata [22]; the latter were studied independently of Greibach's work by Mitrana and Stiebe [25].

Recently, a number of authors have studied connections between the structural properties of a given group $G$ and of the collection of groups whose word problems are recognised by $G$-automata $[9,10,13,22$, 24]. The second author [23, building upon previous work of Elston and the third author [10], has recently given a complete characterisation of the groups whose word problems are accepted by deterministic $G$-automata, for each group $G$.

Theorem 2 (Kambites 2006 [23]). Let $G$ and $H$ be groups, with $H$ finitely generated. Then $H$ has word problem accepted by a deterministic $G$-automaton if and only if $H$ has a finite index subgroup which embeds in $G$.

An interesting question is that of the extent to which this result can be extended to the non-deterministic case. A well-known theorem of Muller and Schupp [26], when combined with a subsequent result of Dunwoody [7], says that a group has context-free word problem if and only if it is virtually free. If $G$ is a free group of rank 2 or more than the $G$-automata accept exactly the context-free languages [3, 5, 22. Moreover, every subgroup of $G$ is free, and $G$ contains every countable rank free group as a subgroup. It follows that the result of Muller and Schupp admits the following equivalent formulation, as a generalisation of Theorem 2 to non-deterministic automata, in the special case that the register group $G$ is free and non-abelian.

Theorem 3 (Muller and Schupp 1983 [26], Dunwoody 1985 [7]). Let G be a free group of rank 2 or more, and $H$ a finitely generated group. Then the word problem for $H$ is accepted by a G-automaton if and only if $H$ has a finite index subgroup which embeds in $G$.

As a consequence of Theorem 1, we deduce the following result, which gives a complete non-deterministic analogue of Theorem 2 in the case that the register group $G$ is virtually abelian, and hence also an abelian analogue of Theorem 3 . 
Theorem 4. Let $G$ be a virtually abelian group and $H$ a finitely generated group. Then $H$ has word problem accepted by a $G$-automaton if and only if $H$ has a finite index subgroup which embeds in $G$.

This generalises and was inspired by the following recent result of Cleary and the first and third authors [4].

Theorem 5 (Cleary, Elder and Ostheimer 2006 [4]). The word problem for $\mathbb{Z}^{p}$ is recognised by a $\mathbb{Z}^{q}$-automaton only if and only if $p \leq q$.

While Theorem 5 was proved directly using only elementary linear algebra, the more general Theorems 1 and 4 seem to require a more complex argument involving growth of groups and the structure of rational sets in $\mathbb{Z}^{n}$.

In addition to this introduction, this paper comprises six sections. Section 2 briefly recalls some definitions and notation which we shall use in the rest of the paper. Section 3 obtains some bounding results concerning minimal elements of intersections of semilinear sets in $\mathbb{Z}^{n}$; these may be of some independent interest. In Section 4 , these bounds are applied to prove that a group with word problem accepted by a $\mathbb{Z}^{n}$-automaton has growth bounded above by a polynomial of degree $n$; it follows by Gromov's polynomial growth theorem [16] that such a group is virtually nilpotent. In Section 5 we use a combinatorial result of Mitrana and Stiebe [25] to show that a virtually nilpotent group with word problem accepted by a $\mathbb{Z}^{n}$-automaton must in fact be virtually abelian. In Section [6 we combine the results of Sections 4 and 5 to prove Theorems 1 and 4. Finally, in Section 7, we consider groups whose word problems are accepted by other kinds of counter automata.

\section{Preliminaries and Notation}

In this section we recall some basic definitions which we shall need in the rest of the paper. We assume a basic familiarity with formal languages, with finite automata over monoids (see [8] for an introduction) and with elementary group theory (see, for example, [28]). Throughout this paper, we write $\mathbb{N}$ for the set of non-negative integers, including 0 . We apply functions on the right of their arguments, and compose them from left to right. We denote by the empty word by $\epsilon$.

Let $G$ be a finitely generated group. Recall that a (finite) choice of generators for $G$ is a surjective monoid morphism $\sigma$ from a (finitely generated) free monoid $X^{*}$ onto $G$. The choice of generators is called symmetric if $X$ comes equipped with a fixed-point-free involution $x \mapsto x^{-1}$ such that $\left(x^{-1}\right) \sigma=(x \sigma)^{-1}$ for all $x \in X$; such an involution extends naturally to the whole of $X^{*}$ by $\left(x_{1} \ldots x_{n}\right)^{-1}=x_{n}^{-1} \ldots x_{1}^{-1}$. We say that a word $w \in X^{*}$ is a representative for the element $w \sigma \in G$. We shall often leave the morphism $\sigma$ implicit, referring to $X$ as a choice of generators for $G$. Recall that the word problem for $G$ with respect to $\sigma$ is the language of all words $w \in X^{*}$ such that $w \sigma=1$ in $G$.

Now suppose we have fixed a choice of generators $X$ for $G$. For each $g \in G$, we define the length of $g$, denoted $|g|$, to be the length of the shortest representative for $g$ in $X^{*}$; if the choice of generators is symmetric then we 
have $\left|g^{-1}\right|=|g|$. For $n \in \mathbb{N}$, the ball of radius $n$ in $G$ is the set

$$
B_{n}(G)=\{g \in G|| g \mid \leq n\} .
$$

Recall that the growth function of $G$ (with respect to $X$, which we usually leave implicit) is the function

$$
\mathbb{N} \rightarrow \mathbb{N}, n \mapsto\left|B_{n}(G)\right|
$$

while the growth of $G$ is the asymptotic complexity class of the growth function. The latter is easily seen to be independent of the choice of generators.

We now recall the definition of an $M$-automaton. Let $M$ be a monoid with identity 1 , and $X$ be a finite alphabet. By an $M$-automaton $A$ over $X$, we mean a finite automaton $A$ over the direct product monoid $M \times X^{*}$. We say that $A$ accepts a word $w \in X^{*}$ if it accepts $(1, w) \in M \times X^{*}$ when considered as a finite automaton in the usual sense, that is, if there is a path from the start state to a terminal state labelled $(1, w)$. The language recognised or accepted by $A$ is the set of all words $w \in X^{*}$ which are accepted by $A$. For a more detailed introduction to $M$-automata, see [12] or [22].

We shall be particularly interested in the case in which the monoid $M$ is actually a group $G$, and the alphabet $X$ is a (typically symmetric) monoid generating set for another group $H$. We shall need the following elementary proposition.

Proposition 1. Let $G$ be a group, and let $H$ be a finitely generated subgroup of a finitely generated group $K$. If the word problem for $K$ (with respect to any choice of generators) is accepted by a G-automaton then the word problem for $H$ (with respect to any choice of generators) is accepted by a $G$-automaton. If $H$ has finite index in $K$ then the converse holds.

Proof. Let $\sigma: X^{*} \rightarrow H$ and $\tau: Y^{*} \rightarrow K$ be finite choices of generators for $H$ and $K$ respectively. For each $x \in X$ choice a word $w_{x} \in Y^{*}$ such that $w_{x} \tau=x \sigma$, and define a morphism $\rho: X^{*} \rightarrow Y^{*}$ by $x \rho=w_{x}$. It is readily verified that the word problem for $H$ with respect to $\sigma$ is the inverse image under $\rho$ of the word problem for $K$ with respect to $\tau$. Since the class of languages accepted by $G$-automata is closed under rational transduction [12, Theorem 6.2], and hence under inverse morphism, this suffices to prove the direct implication.

Conversely, suppose the word problem for $H$ is accepted by a $G$-automaton, and $H$ has finite index in $K$. By [10, Theorem 7], the word problem for $K$ is accepted by a $H$-automaton, and it follows by [22, Corollary 3] that the word problem for $K$ is accepted by a $G$-automaton.

Note that, since a group is a finite index subgroup of itself, Proposition 1 implies in particular that recognisability of the word problem for $H$ by a $G$-automaton is independent of the choice of generators for $H$.

Corollary 1. If the word problem for $H$ with respect to any choice of generators is accepted by a $G$-automaton, then the word problem for $H$ with respect to every choice of generators is accepted by a G-automaton.

We can prove also a similar result for subgroups of the register group. 
Proposition 2. Let $G$ be a group, and $H$ a subgroup of $G$. Then any language accepted by an $H$-automaton is accepted by a $G$-automaton. If $H$ has finite index in $G$ then the converse holds.

Proof. It follows straight from the definitions that any $H$-automaton can be viewed as a $G$-automaton accepting the same language, so the direct implication is immediate.

For the converse, suppose $H$ has finite index in $G$ and that $L$ is accepted by a $G$-automaton. By [10, Theorem 7], the word problem for $G$ is accepted by an $H$-automaton, and so by [22, Corollary 3], the language $L$ is accepted by an $H$-automaton, as required.

\section{Some Bounds for Linear Maps and Semilinear Sets}

The main aim of this section is to establish a bound on the size of the smallest element of the intersection of two semilinear sets, in terms of certain parameters of those sets.

Let $n$ be a non-negative integer. We consider the group $\mathbb{Z}^{n}$ as an additive subgroup of the row vector space $\mathbb{Q}^{n}$; hence we use additive notation. Let $X$ be the set of free generators for $\mathbb{Z}^{n}$ together with their inverses, that is, the set of row vectors with a single non-zero component, which takes the value 1 or -1 . The length function for $\mathbb{Z}^{n}$ with respect to $X$ is the restriction to $\mathbb{Z}^{n}$ of the "Manhattan taxi" norm on $\mathbb{Q}^{n}$, given by

$$
\left|\left(x_{1}, x_{2}, \ldots, x_{n}\right)\right|=\left|x_{1}\right|+\left|x_{2}\right|+\cdots+\left|x_{n}\right|
$$

where $\left|x_{i}\right|$ for $x_{i} \in Q$ denotes the absolute value of $x_{i}$.

Recall that a linear set in $\mathbb{Z}^{n}$ is a set of the form

$$
\left\{s_{0}+\lambda_{1} s_{1}+\cdots+\lambda_{p} s_{p} \mid \lambda_{1}, \ldots, \lambda_{p} \in \mathbb{N}\right\}
$$

for some vectors $s_{0}, s_{1}, \ldots, s_{p} \in \mathbb{Z}^{n}$. A semilinear set is a finite union of linear sets. A theorem of Parikh [27] states that every context-free subset of $\mathbb{Z}^{n}$, and in particular every rational subset of $\mathbb{Z}^{n}$, is in fact semilinear.

In order to prove the main theorem, we will need some results concerning linear maps and semilinear sets in $\mathbb{Z}^{n}$. We begin with the following elementary linear algebraic proposition concerning linear maps.

Proposition 3. Let $\sigma: \mathbb{Z}^{p} \rightarrow \mathbb{Z}^{n}$ be a linear map. Then there exist constants $L, M>0$ such that if $v \in \mathbb{Z}^{n}$ lies in $\left(\mathbb{N}^{p}\right) \sigma$, then $v=u \sigma$ for some $u \in \mathbb{N}^{p}$ with $|u|<L|v|+M$

Proof. Consider first the case in which $\sigma$ is injective. It is readily verified that $\sigma$ extends uniquely to a linear map $\tau: \mathbb{Q}^{p} \rightarrow \mathbb{Q}^{n}$ which is also injective. Now $\tau$ has an inverse $\tau^{-1}: \operatorname{Im} \tau \rightarrow \mathbb{Q}^{p}$, which is also linear. Hence, there exist vectors $x_{1}, \ldots, x_{n} \in \mathbb{Q}^{p}$ such that $v \tau^{-1}=v_{1} x_{1}+\cdots+v_{n} x_{n}$ for every $v \in \operatorname{Im} \tau$, where of course $v_{1}, \ldots, v_{n}$ denote the components of $v$. Let $L$ be the greatest norm of any of the vectors $x_{i}$, and let $M=0$. 
Now suppose $v \in \mathbb{Z}^{n}$ lies in $\mathbb{N}^{p} \sigma$. Then letting $u=v \tau^{-1}$, since $\tau$ is injective, we must have $u \in \mathbb{N}^{p}$. Now using the triangle inequality we have:

$$
\begin{aligned}
|u| & =\left|v \tau^{-1}\right| \\
& =\left|v_{1} x_{1}+\cdots+v_{n} x_{n}\right| \\
& \leq\left|v_{1}\right|\left|x_{1}\right|+\cdots+\left|v_{n}\right|\left|x_{n}\right| \\
& \leq L\left(\left|v_{1}\right|+\cdots+\left|v_{n}\right|\right) \\
& =L|v|+M
\end{aligned}
$$

as required.

For the case in which $\sigma$ is not injective, we proceed by induction on the dimension $p$ of the domain of $\sigma$. First, observe that since every map with domain $\mathbb{Z}^{0}$ is injective, the base case $p=0$ is already proven.

Now let $p \geq 1$, and suppose the claim holds for lesser values of $p$. It is easily checked that, since $\sigma$ is not injective, we can choose a non-zero vector $z \in \mathbb{Z}^{p}$ which lies in the kernel of $\sigma$; by negating $z$ if necessary, we may assume that at least one component of $z$ is positive. Let $c$ be the largest positive component of $z$. Let $x_{1}, \ldots, x_{p} \in \mathbb{Z}^{n}$ be vectors such that

$$
u \sigma=u_{1} x_{1}+\cdots+u_{p} x_{p}
$$

for all $u=\left(u_{1}, \ldots, u_{p}\right) \in \mathbb{Z}^{p}$. For $1 \leq i \leq p$, define

$$
\sigma_{i}: \mathbb{Z}^{p-1} \rightarrow \mathbb{Z}^{n}
$$

by currying $\sigma$ with 0 in the $i$ th position, that is, by

$$
\left(x_{1}, \ldots, x_{i-1}, x_{i+1}, \ldots, x_{p}\right) \sigma_{i}=\left(x_{1}, \ldots x_{i-1}, 0, x_{i+1}, \ldots, x_{p}\right) \sigma .
$$

For each $\sigma_{i}$, let $L_{i}$ and $M_{i}$ be the constants given by the inductive hypothesis, and let $L$ and $M^{\prime}$ be the greatest of the $L_{i}$ s and $M_{i}$ s respectively. Let $q$ be the greatest magnitude of any of the vectors $x_{i}$.

Now suppose $u \sigma=v$ where $u \in \mathbb{N}^{p}$. Then $(u-z) \sigma=v$, and either $(u-z) \in \mathbb{N}^{p}$ or $u_{i}<c$ for some $i$. By subtracting $z$ as many times as possible while remaining in $N^{p}$, we may assume the latter. Now we have

$$
v=u \sigma=u_{1} x_{1}+\cdots+u_{p} x_{p}
$$

so that

$$
\begin{aligned}
v-u_{i} x_{i} & =u_{1} x_{1}+\cdots+u_{i-1} x_{i-1}+u_{i+1} x_{i+1}+\cdots+u_{p} x_{p} \\
& =\left(u_{1}, \ldots, u_{i-1}, u_{i+1}, \ldots, u_{p}\right) \sigma_{i}
\end{aligned}
$$

By the inductive hypothesis, there exists a vector

$$
\left(u_{1}^{\prime}, \ldots, u_{i-1}^{\prime}, u_{i+1}^{\prime}, \ldots, u_{p}^{\prime}\right) \in \mathbb{N}^{p-1}
$$

such that

and

$$
v-u_{i} x_{i}=\left(u_{1}^{\prime}, \ldots, u_{i-1}^{\prime}, u_{i+1}^{\prime}, \ldots, u_{p}^{\prime}\right) \sigma_{i}
$$

$$
u_{1}^{\prime}+\cdots+u_{i-1}^{\prime}+u_{i+1}^{\prime}+\cdots+u_{p}^{\prime}<L_{i}\left|v-u_{i} x_{i}\right|+M_{i} .
$$

Letting

we have

$$
u^{\prime}=\left(u_{1}^{\prime}, \ldots, u_{i-1}^{\prime}, u_{i}, u_{i+1}^{\prime}, \ldots, u_{p}^{\prime}\right)
$$

$$
u^{\prime} \sigma=\left(u_{1}^{\prime}, \ldots, u_{i-1}^{\prime}, u_{i+1}^{\prime}, \ldots, u_{p}^{\prime}\right) \sigma_{i}+u_{i} x_{i}=v .
$$


Moreover, by the triangle inequality, we have

$$
\left|v-u_{i} x_{i}\right| \leq|v|+\left|-u_{i} x_{i}\right|=|v|+u_{i}\left|x_{i}\right| .
$$

which combined with (10) yields

$$
u_{1}^{\prime}+\cdots+u_{i-1}^{\prime}+u_{i+1}^{\prime}+\cdots+u_{p}^{\prime}<L_{i}|v|+L_{i} u_{i}\left|x_{i}\right|+M_{i} .
$$

Letting $u_{i}^{\prime}=u_{i}<c$ and recalling that $L_{i} \leq L, M_{i} \leq M^{\prime}$ and $\left|x_{i}\right| \leq q$ we get

$$
\left|u^{\prime}\right|=u_{1}^{\prime}+\cdots+u_{p}^{\prime} \leq L|v|+L c q+M^{\prime}+c .
$$

Since $L, M^{\prime}, c$ and $q$ are constants chosen independently of $v$, setting $M=$ $L c q+M^{\prime}+c$ suffices to complete the proof.

We can apply Proposition 3 to obtain a related bound on the size of the smallest element of an intersection of linear sets in $\mathbb{Z}^{n}$.

Theorem 6. Let $n, p, q \geq 0$ and suppose $s_{1}, \ldots s_{p}, t_{1}, \ldots t_{q} \in \mathbb{Z}^{n}$. Then there exist constants $P$ and $Q$ such that for any $s_{0}, t_{0} \in \mathbb{Z}^{n}$ the linear sets

$$
S=\left\{s_{0}+\lambda_{1} s_{1}+\cdots+\lambda_{p} s_{p} \mid \lambda_{1}, \ldots, \lambda_{p} \in \mathbb{N}\right\}
$$

and

$$
T=\left\{t_{0}+\mu_{1} t_{1}+\cdots+\mu_{q} t_{q} \mid \mu_{1}, \ldots, \mu_{q} \in \mathbb{N}\right\}
$$

either do not intersect, or have a common element of magnitude less than $P\left(\left|s_{0}\right|+\left|t_{0}\right|\right)+Q$.

Proof. Define a function $\pi: \mathbb{Z}^{p+q} \rightarrow \mathbb{Z}^{n}$ by

$$
\left(\lambda_{1}, \ldots, \lambda_{p}, \mu_{1}, \ldots, \mu_{q}\right) \pi=\lambda_{1} s_{1}+\cdots+\lambda_{p} s_{p}-\mu_{1} t_{1}-\cdots-\mu_{q} t_{q} .
$$

Clearly $\pi$ is linear. Let $L$ and $M$ be the constants given for $\pi$ by Proposition 3. Let $c$ be the greatest magnitude of any of the vectors $s_{i}$. Let $P=L c+1$ and $Q=c M$.

Now suppose $S$ and $T$ intersect, and choose $v \in S \cap T$. Then there exist $\lambda_{1}, \ldots, \lambda_{p}, \mu_{1}, \ldots, \mu_{q} \in \mathbb{N}$ such that

$$
v=s_{0}+\lambda_{1} s_{1}+\cdots+\lambda_{p} s_{p}=t_{0}+\mu_{1} t_{1}+\cdots+\mu_{q} t_{q} .
$$

Now we have

$$
\left(\lambda_{1}, \ldots, \lambda_{p}, \mu_{1}, \ldots, \mu_{p}\right) \pi=\lambda_{1} s_{1}+\cdots+\lambda_{p} s_{p}-\mu_{1} t_{1}-\cdots-\mu_{q} t_{q}=t_{0}-s_{0} .
$$

so that $t_{0}-s_{0}$ lies in the image of the function $\pi$. Now by Proposition 3 , there exist $\lambda_{1}^{\prime}, \ldots, \lambda_{p}^{\prime}, \mu_{1}^{\prime}, \ldots, \mu_{q}^{\prime} \in \mathbb{N}$ such that

$$
\lambda_{1}^{\prime}+\cdots+\lambda_{p}^{\prime}+\mu_{1}^{\prime}+\cdots+\mu_{q}^{\prime}<L\left|t_{0}-s_{0}\right|+M
$$

and

$$
\left(\lambda_{1}^{\prime}, \ldots, \lambda_{p}^{\prime}, \mu_{1}^{\prime}, \ldots, \mu_{q}^{\prime}\right) \pi=t_{0}-s_{0}
$$

Using the definition of $\pi$, it follows that the element

$$
v^{\prime}=s_{0}+\lambda_{1}^{\prime} s_{1}+\cdots+\lambda_{p}^{\prime} s_{p}=t_{0}+\mu_{1}^{\prime} t_{1}+\cdots+\mu_{q}^{\prime} t_{q} .
$$

lies in $S \cap T$. Moreover, using the triangle inequality, we have

$$
\begin{aligned}
\left|v^{\prime}\right| & =\left|s_{0}+\lambda_{1}^{\prime} s_{1}+\cdots+\lambda_{p}^{\prime} s_{p}\right| \\
& \leq\left|s_{0}\right|+\lambda_{1}^{\prime}\left|s_{1}\right|+\cdots+\lambda_{p}^{\prime}\left|s_{p}\right| \\
& \leq\left|s_{0}\right|+\left(\lambda_{1}^{\prime}+\cdots+\lambda_{p}^{\prime}\right) c
\end{aligned}
$$


Now by (2), we have

$$
\lambda_{1}^{\prime}+\cdots+\lambda_{p}^{\prime} \leq \lambda_{1}^{\prime}+\cdots+\lambda_{p}^{\prime}+\mu_{1}^{\prime}+\cdots+\mu_{q}^{\prime} \leq L\left(\left|t_{0}-s_{0}\right|\right)+M
$$

so that

$$
\begin{aligned}
\left|v^{\prime}\right| & \leq\left|s_{0}\right|+L c\left|t_{0}-s_{0}\right|+c M \\
& \leq\left|s_{0}\right|+L c\left|t_{0}\right|+L c\left|s_{0}\right|+c M \quad \text { (by the triangle inequality) } \\
& \leq(L c+1)\left(\left|s_{0}\right|+\left|t_{0}\right|\right)+c M \\
& =P\left(\left|s_{0}\right|+\left|t_{0}\right|\right)+Q
\end{aligned}
$$

as required.

Corollary 2. Let $R$ be a finite set of vectors in $\mathbb{Z}^{n}$. Then there exist positive integers $P$ and $Q$ such that any linear sets

$$
S=\left\{s_{0}+\lambda_{1} s_{1}+\cdots+\lambda_{p} s_{p} \mid \lambda_{1}, \ldots, \lambda_{p} \in \mathbb{N}\right\}
$$

and

$$
T=\left\{t_{0}+\mu_{1} t_{1}+\cdots+\mu_{q} t_{q} \mid \mu_{1}, \ldots, \mu_{q} \in \mathbb{N}\right\}
$$

with $s_{1}, \ldots, s_{p}, t_{1}, \ldots t_{q} \in R$ either do not intersect, or have a common element of magnitude less than $P\left(\left|s_{0}\right|+\left|t_{0}\right|\right)+Q$.

Proof. Let $R^{\prime}$ denote the (finite) set of all finite sequences of elements from $R$ which do not contain the same element twice. For each pair of sequences $s=\left(s_{1}, \ldots, s_{p}\right) \in R^{\prime}$ and $t=\left(t_{1}, \ldots, t_{q}\right) \in R^{\prime}$, let $P_{s t}$ and $Q_{s t}$ be the values given by Theorem 6, and choose $P$ and $Q$ to exceed all the $P_{s t}$ and $Q_{s t}$ respectively.

Now let $S$ and $T$ be as given in the statement, and suppose that they intersect. Let $s=\left(s_{1}, \ldots, s_{p}\right)$ and $t=\left(t_{1}, \ldots, t_{q}\right)$. Clearly, we may assume without loss of generality that $s_{i} \neq s_{j}$ for $1 \leq i<j \leq p$ so that $s \in R^{\prime}$, and similarly $t \in R^{\prime}$. By Theorem 6, $S$ and $T$ have a common element of norm less than $P_{s t}\left(\left|s_{0}\right|+\left|t_{0}\right|\right)+Q_{s t}$, which in turn is less than $P\left(\left|s_{0}\right|+\left|t_{0}\right|\right)+Q$, as required.

We now introduce some terminology for certain parameters of linear and semilinear sets of $\mathbb{Z}^{n}$. Let $p$ be a non-negative integer. We say that a linear subset $S$ of $\mathbb{Z}^{n}$ has constant bound $p$ if there exist $s_{0}, \ldots, s_{q} \in G$ with $\left|s_{0}\right| \leq p$ such that

$$
S=\left\{s_{0}+\lambda_{1} s_{1}+\cdots+\lambda_{q} s_{q} \mid \lambda_{1}, \ldots, \lambda_{q} \in \mathbb{N}\right\} .
$$

Similarly, $S$ has generator bound $p$ if there exist $s_{0}, \ldots, s_{q} \in G$ satisfying the above equation with $\left|s_{i}\right| \leq p$ for $1 \leq i \leq q$. A semilinear set has constant bound $p$ [respectively, generator bound $p$ ], if it is a finite union of linear sets with constant bound $p$ [generator bound $p$ ]. We state two elementary properties of these parameters, which follow directly from the definitions.

Proposition 4. Let $S$ be a linear [semilinear] subset of $\mathbb{Z}^{n}$ with constant bound $P$ and generator bound $Q$. Then the set

$$
-S=\{-s \mid s \in S\}
$$

is a linear [semilinear] set with constant bound $P$ and generator bound $Q$. 
Proposition 5. Suppose $S$ and $T$ are linear [semilinear] sets with constant bounds $P$ and $Q$ respectively, and generator bounds $R$ and $S$ respectively. Then the set

$$
S+T=\{s+t \mid s \in S, t \in T\}
$$

is a linear [semilinear] set with constant bound $P+Q$ and generator bound $\max (R, S)$.

Theorem 7. Let $K$ be a positive integer. Then there exist positive integers $C$ and $D$ such that for any semilinear sets $S$ and $T$ in $\mathbb{Z}^{n}$ with constant bound $m$ and generator bound $K$, either $S$ and $T$ do not intersect, or they have a common element of magnitude less than $C m+D$.

Proof. Let $R$ be the (finite) set of all vectors in $\mathbb{Z}^{n}$ of norm less than $K$, and let $P$ and $Q$ be the values given by Corollary 2 , Let $C=2 P$ and $D=Q$.

Now suppose $S$ and $T$ are intersecting semilinear sets with constant bound $m$ and generator bound $K$. Then each is a finite union of linear components with constant bound $m$ and generator bound $K$, where some component $S^{\prime}$ of $S$ intersects with some component $T^{\prime}$ of $T$. Writing

$$
S^{\prime}=\left\{s_{0}+\lambda_{1} s_{1}+\cdots+\lambda_{p} s_{p} \mid \lambda_{1}, \ldots, \lambda_{p} \in \mathbb{N}\right\}
$$

and

$$
T^{\prime}=\left\{t_{0}+\mu_{1} t_{1}+\cdots+\mu_{q} t_{q} \mid \mu_{1}, \ldots, \mu_{q} \in \mathbb{N}\right\},
$$

by Corollary 2 there is an element in $S^{\prime} \cap T^{\prime}$, and hence in $S \cap T$, with norm less than

$$
P\left(\left|s_{0}\right|+\left|t_{0}\right|\right)+Q \leq P(m+m)+Q=2 P m+Q=C m+D .
$$

\section{The Growth of Counter Groups}

In this section, we apply the main result of Section 3 to show that a group with word problem accepted by a $\mathbb{Z}^{n}$-automaton has growth bounded above by a polynomial of degree $n$. It follows by Gromov's polynomial growth theorem [16] that such a group is virtually nilpotent; in Sections 5 and 6 we shall see that every such group is in fact virtually abelian.

Theorem 8. Let $H$ be a group. If the word problem for $H$ is accepted by a $\mathbb{Z}^{n}$-automaton then $H$ has growth bounded above by a polynomial of degree $n$.

Proof. Fix a finite, symmetric choice of generators $X$ for $H$; then by Corollary 11 we may let $A$ be a $\mathbb{Z}^{n}$-automaton accepting the word problem $W_{X}(H)$ of $H$ with respect to $X$. Clearly, we may assume without loss of generality that every edge in $A$ has label of the form $(g, x)$ for some $g \in \mathbb{Z}^{n}$ and $x \in X \cup\{\epsilon\}$.

To prove the lemma, it will suffice to demonstrate the existence of a function $\sigma: H \rightarrow \mathbb{Z}^{n}$ (which need not be a morphism) and constants $P, Q$ and $R$ such that:

(i) the pre-image $g \sigma^{-1}$ of each element $g \in \mathbb{Z}^{n}$ contains at most $R$ elements; and

(ii) for any $h \in H$, we have $|h \sigma| \leq P|h|+Q$. 
Indeed, suppose $\sigma, P, Q$ and $R$ satisfy these properties. Then clearly, $\left|B_{n}(H)\right| \leq R\left(\left|B_{P n+Q}\left(\mathbb{Z}^{n}\right)\right|\right)$ for all $n$. Since the growth function of $\mathbb{Z}^{n}$ is a polynomial of degree $n$, and the composition of a polynomial of degree $n$ with a linear function is a polynomial of degree $n$, this suffices to show that the growth of $H$ is bounded above by a polynomial of degree $n$, as required. The rest of this section, then, is concerned with finding a function $\sigma$ and constants $P, Q$ and $R$ with the above properties.

We begin with a definition. For each pair of states $p, q$ in the automaton $A$, let $R_{p q}$ denote the set of all element $g \in \mathbb{Z}^{n}$ such that $(g, \epsilon)$ labels a path from $p$ to $q$. By removing from $A$ all edges with label of the form $(g, w)$ for $w \neq \epsilon$ and then ignoring the second components of the edge labels, we can clearly obtain a finite automaton over $\mathbb{Z}^{n}$ which, with initial state $p$ and terminal state $q$ accepts $R_{p q}$. Thus, each $R_{p q}$ is a rational subset of $\mathbb{Z}^{n}$, and so by Parikh's theorem [27] is semilinear.

Since there are finitely many sets of the form $R_{p q}$, there clearly exist constants $F$ and $K$ such that every $R_{p q}$ has constant bound $F$ and generator bound $K$. Moreover, since $A$ has only finitely many edges, we can choose $F$ also to be greater than any $|g|$ such that $A$ has an edge with label of the form $(g, x)$. Let $C$ and $D$ be the constants given by Theorem 7 for our chosen value of $K$. Let $P=2 C F$ and $Q=C F+D$. Let $R$ be the number of states in the automaton $A$.

The main work of the proof is done in three lemmas.

Lemma 1. Let $w \in X^{*}$, and suppose the automaton $A$ has a path from $p$ to $q$ labelled $(g, w)$. Then there exists a subset $S \subseteq \mathbb{Z}^{n}$ such that

(i) $S$ is linear with constant bound $(2|w|+1) F$ and generator bound $K$;

(ii) $g \in S$;

(iii) for every $s \in S,(s, w)$ labels a path from $p$ to $q$ in $A$.

Proof. Suppose $w=w_{1} \ldots w_{m}$ with each $w_{i} \in X$, so that $m=|w|$. Let $\pi$ be a path from $p$ to $q$ labelled $(g, w)$. It follows from our assumption above about the edge labels in $A$ that the path $\pi$ can be decomposed as

$$
\pi=p_{0} e_{1} p_{1} e_{2} \ldots e_{m} p_{m}
$$

where

- each $p_{i}$ is a path with label $\left(k_{i}, \epsilon\right)$ for some $k_{i} \in \mathbb{Z}^{n}$, and

- each $e_{i}$ is an edge with label $\left(g_{i}, w_{i}\right)$ for some $g_{i} \in \mathbb{Z}^{n}$.

where of course $k_{0}+g_{1}+k_{1}+\cdots+g_{m}+k_{m}=g$.

We define

$$
T=\left\{g_{1}\right\}+\cdots+\left\{g_{m}\right\}+R_{p_{0} \alpha, p_{0} \omega}+\cdots+R_{p_{m} \alpha, p_{m} \omega}
$$

where $p_{i} \alpha$ and $p_{i} \omega$ denote respectively the start state and the end state of the path $p_{i}$. It is immediate from the definitions that each element $k_{i}$ lies in the semilinear set $R_{p_{i} \alpha, p_{i} \omega}$. Hence, we see that $g=k_{0}+g_{1}+k_{1}+\cdots+g_{n}+k_{n}$ lies in $T$.

Notice that $T$ is the sum of $2 m+1$ semilinear sets each of which has constant bound $F$ and generator bound $K$. It follows by Proposition 5 that $T$ is a semilinear set with constant bound $(2 m+1) F$ and generator bound $K$. But this means that $T$ is a finite union of linear sets with constant bound $(2 m+1) F$ and generator bound $K$, at least one of which must contain $k$; 
choose $S$ to be such a set. Finally, it follows immediately from the definition of the sets $R_{p q}$ that for every element of $s \in T$, and hence certainly for every element $s \in S$, we have that $(s, w)$ labels a path from $p$ to $q$, as required.

Lemma 2. Let $w \in X^{*}$. Then there exists a state $q$ in $A$ and an element $g \in G$ with $|g|<P|w|+Q$ such that $(g, w)$ labels a path from the initial state to $q$, and $\left(-g, w^{-1}\right)$ labels a path from $q$ to some terminal state.

Proof. Certainly $w w^{-1} \in X^{*}$ lies in the word problem of $H$ and so is accepted by the $\mathbb{Z}^{n}$-automaton $A$. It follows that there is an element $k \in \mathbb{Z}^{n}$ and a vertex $q$ in $A$, such that $A$ has

- a path $\pi_{1}$ from the initial state to $q$ labelled $(k, w)$; and

- a path $\pi_{2}$ from $q$ to a terminal state labelled $\left(-k, w^{-1}\right)$.

By Lemma 1, there exist linear sets $S$ and $T$, each with constant bound $(2|w|+1) F=\left(2\left|w^{-1}\right|+1\right) F$ and generator bound $K$, such that

- $k \in S$;

- $-k \in T$;

- for every $s \in S$, there is a path from the initial state to $q$ labelled $(s, w)$;

- for every $t \in T$, there is a path from $q$ to a terminal state labelled $\left(t, w^{-1}\right)$

By Proposition 4, the set $-T$ is also linear with constant bound $(2|w|+1) F$ and generator bound $K$. Moreover, $k \in S \cap(-T)$. Hence, by Theorem 7 and the choice of $C$ and $D$ above, there exists an element $g \in S \cap(-T)$ with

$$
|g|<C(2|w|+1) F+D=2 C F|w|+C F+D=P|w|+Q .
$$

Now $g \in S$ and $-g \in T$, so that $g$ has the required properties.

Lemma 3. Let $g \in \mathbb{Z}^{n}$, and let $S \subseteq X^{*}$ be such that for every $w \in S$, there exists a state $q_{w}$ in $A$ such that $(g, w)$ labels a path from the initial state to $q_{w}$, and $\left(-g, w^{-1}\right)$ labels a path from $q_{w}$ to a terminal state. Then $S$ contains representatives for at most $R$ elements of $H$.

Proof. Suppose for a contradiction that $S$ has the given properties, but contains representatives for strictly more than $R$ elements of $H$. Then by the pigeon hole principle, since $A$ has only $R$ states, there exist words $u, v \in S$ such that $u$ and $v$ represent distinct elements of $H$, but $q_{u}=q_{v}$. But now there is a path from the initial state to $q_{u}$ labelled $(g, u)$, and a path from $q_{u}=q_{v}$ to a terminal state labelled $\left(-g, v^{-1}\right)$. Hence, the $\mathbb{Z}^{n}$-automaton $A$ accepts the word $u v^{-1}$, so we must have that $u v^{-1}$ lies in the word problem for $H$. But this means that $u$ and $v$ represent the same element of $H$, giving the desired contradiction.

We are now ready to define our function $\sigma: H \rightarrow \mathbb{Z}^{n}$. For each $h \in H$, we choose some word $w \in X^{*}$ of minimal length representing $h$. Lemma 2 guarantees the existence of at least one element $g$ with $|g|<P|w|+Q=$ $P|h|+Q$ such that $(g, w)$ labels a path from an initial state to some state $q$, and $\left(-g, w^{-1}\right)$ labels a path from $q$ to a terminal state. We choose $h \sigma$ to be one such element. It is immediate that $\sigma$ satisfies the desired bound restriction. Moreover, by Lemma 3, no more than $R$ choices of $h$ can give rise to the same $h \sigma$; indeed otherwise setting $S$ to be the set of all the words 
$w$ chosen for such values of $h$ would contradict Lemma 3, This completes the proof of Theorem 8 .

\section{From Virtually Nilpotent to Virtually Abelian}

The objective of this section is to show that a group which is virtually nilpotent and which has word problem recognised by a blind counter automaton is in fact virtually abelian. Our main tool is the following "interchange lemma" of Mitrana and Stiebe [25].

Lemma 4 (Mitrana and Stiebe 1997 [25]). Let $G$ be an abelian group and $L$ a language. Suppose $L$ is accepted by a $G$-automaton. Then there exists a constant $p$ such that for any word $w \in L$ of length at least $p$, and for any factorisation

$$
w=v_{1} w_{1} v_{2} w_{2} \ldots w_{p} v_{p+1}
$$

with each $\left|w_{i}\right| \geq 1$, there exists integers $r$ and $s$ with $1 \leq r<s \leq p$ such that the word obtained from $w$ by interchanging the factors $w_{r}$ and $w_{s}$ lies in $L$.

We now apply Lemma 4 to prove the main result of this section.

Theorem 9. Suppose $H$ is a finitely generated virtually nilpotent group with word problem accepted by a $\mathbb{Z}^{n}$-automaton. Then $H$ is virtually abelian.

Proof. We claim first that it suffices to prove the result for the case that $H$ is nilpotent and torsion-free. Indeed, if $H$ is virtually nilpotent then let $K$ be a nilpotent subgroup of finite index in $H$. Now by [29, Theorem 5.5] $K$ embeds in $G L(n, \mathbb{Z})$ for some $n$; now by [6, Lemma 9] $K$ has a finite index subgroup $L$ which is torsion-free. Since a subgroup of a nilpotent group is nilpotent, $L$ is also nilpotent. By Proposition 1, the word problem for $L$ is also accepted by a $\mathbb{Z}^{n}$-automaton. If the result holds for torsion-free nilpotent groups we may deduce that $L$ is virtually abelian and hence, since $L$ has finite index in $H$, that $H$ is virtually abelian.

Suppose, then, that $H$ is not abelian, but is torsion free nilpotent with centre $Z$. Choose an element $a$ in the second term of the upper central series for $H$, that is, such that $a$ is not central in $H$ but $a Z$ is central in $H / Z$. Let $b \in H$ be an element which does not commute with $a$. Let $c=[a, b]=$ $a^{-1} b^{-1} a b$. Notice that $c$ is central; indeed $a b Z=a Z b Z=b Z a Z=b a Z$ so that $a b=b a c \in b a Z$, from which it follows that $c \in Z$.

Choose a monoid generating set $X$ for $H$ with elements $x, y$ and $z$ representing to $a, b$ and $c$ respectively. By Corollary 1, the word problem for $H$ with respect to $X$ is accepted by a $\mathbb{Z}^{n}$-automaton. Let $p$ be the interchange constant for $A_{G}$ posited by Lemma 4 .

Let $w=w_{1} \ldots w_{m} \in\{x, y\}^{*}$. We define the exchange index of $w$ to be the number of distinct pairs $(i, j)$ with $1 \leq i<j \leq m$ such that $w_{i}=y$ and $w_{j}=x$; it is the minimum number of times one would have to replace a factor $y x$ by $x y$ in order to obtain a word of the form $x^{k} y^{l}$.

Lemma 5. Let $u, v \in\{x, y\}^{*}$ be words each containing $i$ copies of the letter $x$, and $j$ copies of the letter $y$. If $u$ and $v$ represent the same element of $H$, then they have the same exchange index. 
Proof. Suppose $u$ and $v$ have exchange indices $p$ and $q$ respectively. Since the commutator $c$ of $a$ and $b$ is central, we have

$$
g a b h=g b a c h=(g b a h) c
$$

for all $g, h \in H$. It follows by an easy inductive argument that $u$ represents the element $a^{i} b^{j} c^{p}$ while $v$ represents the element $a^{i} b^{j} c^{q}$. If $u=v$ then we have $c^{p}=c^{q}$ and hence, since $H$ is torsion free, $p=q$ as required.

Consider now the word

$$
t_{1}=x y x y^{2} x y^{3} \ldots x y^{n},
$$

and let $w$ be any word for its inverse, so that $t_{1} w$ lies in the word problem of $H$. Choose $n>p$ and set $v_{i}=x, w_{i}=y^{i}$ for $1 \leq i \leq n$ and $v_{n+1}=u$. Then by Lemma 4 for some $r$ and $s$ we have that

$$
x y x y^{2} x y^{3} \ldots x y^{s} \ldots x y^{r} \ldots x y^{n} w
$$

is also in the word problem for $H$, from which it follows that $t_{1}$ and

$$
t_{2}=x y x y^{2} x y^{3} \ldots x y^{s} \ldots x y^{r} \ldots x y^{n}
$$

both represent the same element of $H$. But $t_{1}$ and $t_{2}$ contain the same number of $x$ s and $y$ s respectively, while a simple calculation shows that their exchange indices differ by $(r-s)^{2}$. This contradicts Lemma 5, and hence completes the proof.

\section{Proofs of the Main Theorems}

Combining the results of Sections 4 and 5 yields our first main result.

Theorem 1. Let $H$ be a finitely generated group. Then the word problem for $H$ is accepted by a blind $n$-counter automaton if and only if $H$ is virtually free abelian of rank $n$ or less.

Proof. Suppose the word problem for $H$ is accepted by a blind $n$-counter automaton, that is, a $\mathbb{Z}^{n}$-automaton. By Theorem 8 , $H$ has growth bounded above by a polynomial of degree $n$. In particular, $H$ has polynomial growth, and so by a famous theorem of Gromov [16] is virtually nilpotent. It follows by Theorem 9 that $H$ is virtually abelian. It follows easily from the structure theorem for finitely generated abelian groups [28, Theorem 4.2.10] that $H$ has a finite index subgroup isomorphic to $\mathbb{Z}^{k}$ for some $k \in \mathbb{N}$. Moreover, the asymptotic growth of $H$ is a polynomial of degree $k$, so we must have $k \leq n$.

Conversely, if $H$ is virtually free abelian of rank $n$ then it has a finite index subgroup isomorphic to $\mathbb{Z}^{n}$; now by [10, Theorem 7], the word problem for $H$ is accepted by a $\mathbb{Z}^{n}$-automaton, that is, a blind $n$-counter automaton, as required.

Theorem 1 leads to our second main result.

Theorem 4. Let $G$ be a virtually abelian group. Then a group $H$ has word problem accepted by a G-automaton if and only if $H$ has a finite index subgroup which embeds in $G$. 
Proof. Suppose first that the word problem for $H$ is accepted by a $G$ automaton. Since $G$ has a finite index abelian subgroup, it follows easily from the structure theorem for finitely generated abelian groups [28, Theorem 4.2.10] that $G$ has a finite index subgroup isomorphic to $\mathbb{Z}^{n}$ for some $n \in \mathbb{N}$. Now by Proposition 2, the word problem for $H$ is accepted by a $\mathbb{Z}^{n}$ automaton, so by Theorem 1, $H$ has a finite index subgroup isomorphic to $\mathbb{Z}^{k}$ for some $k \leq n$. Now $\mathbb{Z}^{k}$ embeds in $\mathbb{Z}^{n}$, which embeds in $G$ as required.

Conversely, if $H$ has a finite index subgroup which embeds in $G$ then by [10, Theorem 7], the word problem for $H$ is accepted by a $G$-automaton.

We also obtain an answer to a question of the second author [23, Question 10] for the class of virtually abelian groups.

Corollary 3. Let $G$ be a virtually abelian group. Then a group word problem is accepted by a deterministic G-automaton if and only if it is accepted by a non-deterministic G-automaton.

Proof. Suppose $H$ has word problem accepted by a non-deterministic $G$ automaton. Then by Theorem 4, $H$ has a finite index subgroup which embeds in $G$. Now by [10, Theorem 7], the word problem for $H$ is recognised by a deterministic $G$-automaton. The converse implication is immediate.

\section{Other Counter Automata}

In this section, we discuss the relationships between blind counter automata, that is, $\mathbb{Z}^{n}$-automata, and other notions of counter automata.

First, we consider the difference between blind and non-blind counter automata. The latter differ from the automata we have studied in that they are endowed with the extra ability to test whether the value of a register is zero; for a formal definition see [17], [18] or [21, Chapter 7]. It is well known that a non-blind automaton with two or more counters can simulate an arbitrary Turing machine [21, Theorem 7.9]. Since, for a counter automaton to accept a language, we do not require that the machine should "terminate" on words not contained in the language, it follows that 2-counter non-blind automata can accept all the recursively enumerable languages and hence, by Higman's embedding theorem [19], word problems of exactly the finitely presented groups and their finitely generated subgroups.

The remaining case of 1-counter non-blind automata was studied by Herbst [17; he showed that a group has word problem accepted by such an automaton if and only if it is virtually cyclic (see also [18]). Combining with Theorem 4, we see that, when attention is restricted to word problems for groups, there is no difference in accepting power between blind and non-blind 1-counter automata.

Corollary 4. A group word problem is accepted by a non-blind 1-counter automaton if and only if it is accepted by a blind 1-counter automaton, that is, a $\mathbb{Z}$-automaton.

The proof of Herbst's result [17, and hence that of Corollary 4, depends upon the Muller-Schupp theorem [26] and thus also on Stallings' theory of ends [30] and the accessibility of finitely presented groups [7]. One is drawn is ask if there is an easier approach. 
Problem 1. Find an elementary proof of Corollary 4.

Such a proof combined with our proof of Theorem 1 above would yield also a purely combinatorial proof of the fact that every finitely generated group with word problem accepted by a non-blind 1-counter automaton has linear growth. Since there is also an elementary proof that the only finitely generated groups of linear growth are the virtually cyclic groups [31], it would also give a straightforward combinatorial proof of the result of Herbst itself.

Another notion of counter automaton has been studied by Cho [2]. We use here a definition easily seen to be equivalent in accepting power to his, although our notation is chosen to make clearer the relationship between his automata and $G$-automata. A $k$-counter Cho automaton over an alphabet $X$ consists of

- a finite automaton over the direct product monoid $\mathbb{N}^{k} \times X^{*}$, with edge labels drawn from $\mathbb{N}^{k} \times X$; and

- for each state $v$ in the automaton, a semilinear subset $S_{v}$ of $\mathbb{N}^{k}$.

A word $w \in X^{*}$ is accepted by the automaton if there is a path from the initial state to some state $v$ with label $(g, w)$, such that $g \in S_{v}$. The automaton is called deterministic if for each state $p$ and letter $x \in X$, there is at most one edge leaving $p$ with label of the form $(g, x)$. For a detailed discussion of these automata, and the languages they accept, see [2]. The following theorem characterises the groups whose word problems are accepted by Cho automata.

Theorem 10. Let $H$ be a finitely generated group. Then the following are equivalent

(i) the word problem for $H$ is accepted by a deterministic Cho automaton;

(ii) the word problem for $H$ is accepted by a Cho automaton;

(iii) $H$ is virtually abelian.

Proof. That (i) implies (ii) is immediate, so suppose that (ii) holds, and let $A$ be a $k$-counter Cho automaton accepting the word problem for $H$. We view $\mathbb{N}^{k}$ as embedded in the natural way into $\mathbb{Z}^{k}$, so that $A$ can be viewed as a $\mathbb{Z}^{k}$-automaton. We construct from $A$ a new $\mathbb{Z}^{k}$-automaton by adding some extra states and edges as follows.

For each state $p$, write the semilinear set $S_{p}$ as a union

$$
S_{p}=S_{p}^{1} \cup S_{p}^{2} \cup \cdots \cup S_{p}^{j}
$$

where each set $S_{p}^{i}$ is linear. For each $i$, let $v_{0}^{i}, v_{1}^{i}, \ldots, v_{m}^{i}$ be such that

$$
S_{p}^{i}=\left\{v_{0}^{i}+\lambda_{1} v_{1}^{i}+\cdots+\lambda_{m} v_{m}^{i} \mid \lambda_{1}, \ldots, \lambda_{m} \in \mathbb{N}\right\}
$$

For each $i$, the new automaton $B$ has an extra state $p_{i}$, an edge from $p$ to $p_{i}$ labelled $\left(-v_{0}^{i}, \epsilon\right)$, and edges from $p_{i}$ to $p_{i}$ labelled $\left(-v_{q}^{i}, \epsilon\right)$ for $1 \leq q \leq m$. The initial state of $B$ is the initial state of $A$, while the terminal states of $B$ are the new states of the form $p_{i}$.

If a word $w$ is accepted by the Cho automaton $B$ then $B$ has a path from the initial state to a some state $p$ labelled $(g, w)$ for some $g \in S_{p}$. Since $A$ contains all the states and edges of $B$, we deduce that $A$ also has a path 
from the initial state to $p$ labelled $(g, w)$. Now $g$ must lie in one of the sets $S_{p}^{i}$. It follows easily that $A$ has a path from $p$ to $p_{i}$ labelled $(-g, \epsilon)$. Thus, $A$ has a path from the initial state to the terminal state $p_{i}$ with label $(1, w)$, so that $A$ accepted $w$.

Conversely, if $A$ accepts a word $w$ then it must have a path from the initial state to some state $p$ labelled $(g, w)$ and then a path from $p$ to some terminal state $p_{i}$ labelled $(-g, \epsilon)$ Clearly the former path must exist also in $B$ and we must have $g \in S_{p}^{i} \subseteq S_{p}$, so that $w$ is also accepted by $B$.

Thus, the $\mathbb{Z}^{k}$-automaton $B$ accepts the same language as the Cho automaton $A$, that is, the word problem for $H$, and so (iii) holds.

Finally, suppose (iii) holds. Then by Theorem 4 and Corollary 3 , $H$ has word problem accepted by a deterministic $\mathbb{Z}^{k}$-automaton $A$ for some $k$. We shall construct from $A$ a deterministic Cho automaton with $2 k$ counters recognising the same language.

Suppose $\mathbb{Z}^{k}$ is generated by

$$
\left\{v_{1}, \ldots, v_{k}\right\}
$$

and $\mathbb{N}^{2 k}$ is generated by

$$
\left\{v_{1}, \bar{v}_{1}, \ldots, v_{k}, \bar{v}_{k}\right\} .
$$

We define a surjective morphism $\sigma: \mathbb{N}^{2 k} \rightarrow Z^{k}$ by $v_{i} \sigma=v_{i}$ and $\bar{v}_{i} \sigma=v_{i}^{-1}$ for $1 \leq i \leq k$. Define a set

$$
S=\left\{\lambda_{1}\left(v_{1}+\bar{v}_{1}\right)+\lambda_{2}\left(v_{2}+\bar{v}_{2}\right)+\cdots+\lambda_{k}\left(v_{k}+\bar{v}_{k}\right) \mid \lambda_{1}, \lambda_{2}, \ldots, \lambda_{k} \in \mathbb{N}\right\} .
$$

Then $S$ is semilinear, and it is easily seen that $S=1 \sigma^{-1}$.

We now define an $2 k$-counter Cho automaton $B$ with the same state set as $A$, the same designated initial state, and edges constructed as follows. For each edge in $A$ from $p$ to $q$ with label $(g, w)$, we choose some element $v \in \mathbb{N}^{2 k}$ with $v \sigma=g$, and give $B$ an edge from $p$ to $q$ with label $(v, w)$. We define $S_{q}=S$ if $q$ is a terminal state in $A$, and $S_{q}=\emptyset$ otherwise.

An easy inductive argument shows that $A$ has a path from $p$ to $q$ with label $(g, w)$ if and only if $B$ has a path from $p$ to $q$ with label $(v, w)$ for some $v$ such that $v \sigma=g$. In particular, $A$ has a path from an initial state to a terminal state with label $(1, w)$ if and only if $B$ has a path from the initial state to a state $q$ with $S_{q}=S$ having label $(v, w)$ for some $v$ with $v \sigma=1$, that is, for some $v \in S$. Hence $w$ is accepted by $A$ if and only if $w$ is accepted by $B$, as required to complete the proof.

Note that Theorem 10 does not quite provide a complete characterisation of groups with word problems recognised by $k$-counter Cho automata for each value of $k$. The proof of Theorem 10 shows only that a group which is virtually free abelian of rank $k$ has word problem accepted by a $2 k$-counter Cho automaton, while a group with word problem accepted by a $k$-counter Cho automaton is virtually free abelian of rank $k$ or less. We conjecture that the former bound is tight while the latter can be strengthened.

Conjecture 1. Suppose $H$ is a group with word problem accepted by a $2 k$ counter or $(2 k+1)$-counter Cho automaton. Then $H$ is virtually free abelian of rank $k$. 


\section{ACKNOWLEDGEMENTS}

This research was started while the second author was at Universität Kassel supported by a Marie Curie Fellowship within the 6th European Community Framework Programme; it was completed while he was supported by an RCUK Academic Fellowship in Manchester. The third author gratefully acknowledges partial support from European Community funds during a visit to Kassel. The authors would like to thank Hong-Ray Cho, Sean Cleary, John Fountain, Bob Gilman, Elaine Render and Benjamin Steinberg for many helpful conversations.

\section{REFERENCES}

[1] W. W. Boone and G. Higman. An algebraic characterization of groups with soluble word problem. J. Austral. Math. Soc., 18:41-53, 1974. Collection of articles dedicated to the memory of Hanna Neumann, IX.

[2] Hong-Ray Cho. An Introduction to Counter Groups. PhD thesis, Stevens Institute of Technology, 2006.

[3] N. Chomsky and M. P. Schützenberger. The algebraic theory of context-free languages. In Computer programming and formal systems, pages 118-161. NorthHolland, Amsterdam, 1963.

[4] S. Cleary, M. Elder, and G. Ostheimer. The word problem distinguishes counter languages. arXiv:math.GR/0606415, 2006.

[5] J. M. Corson. Extended finite automata and word problems. Internat. J. Algebra Comput., 15(3):455-466, 2005.

[6] J. D. Dixon. The Structure of Linear Groups. Van Nostrand Reinhold, New York, 1971.

[7] M. J. Dunwoody. The accessibility of finitely presented groups. Invent. Math., 81(3):449-457, 1985.

[8] S. Eilenberg. Automata, languages, and machines. Vol. A. Academic Press [A subsidiary of Harcourt Brace Jovanovich, Publishers], New York, 1974. Pure and Applied Mathematics, Vol. 58.

[9] M. Elder. G-automata, counter languages and the Chomsky hierarchy. arXiv:math.GR/0508166, 2005.

[10] G. Z. Elston and G. Ostheimer. On groups whose word problem is solved by a counter automaton. Theoret. Comput. Sci., 320(2-3):175-185, 2004.

[11] R. H. Gilman. Groups and languages. Talks given at the City University of New York, October 1999.

[12] R. H. Gilman. Formal languages and infinite groups. In Geometric and computational perspectives on infinite groups (Minneapolis, MN and New Brunswick, NJ, 1994), volume 25 of DIMACS Ser. Discrete Math. Theoret. Comput. Sci., pages 27-51. Amer. Math. Soc., Providence, RI, 1996.

[13] R. H. Gilman and M. Shapiro. On groups whose word problem is solved by a nested stack automaton. arXiv:math.GR/9812028, 1998.

[14] S. A. Greibach. Remarks on the complexity of nondeterministic counter languages. Theoret. Comput. Sci., 1(4):269-288, 1975/76.

[15] S. A. Greibach. Remarks on blind and partially blind one-way multicounter machines. Theoret. Comput. Sci., 7(3):311-324, 1978.

[16] M. Gromov. Groups of polynomial growth and expanding maps. Inst. Hautes Études Sci. Publ. Math., (53):53-73, 1981.

[17] T. Herbst. On a subclass of context-free groups. RAIRO Inform. Théor. Appl., 25(3):255-272, 1991.

[18] T. Herbst and R. M. Thomas. Group presentations, formal languages and characterizations of one-counter groups. Theoret. Comput. Sci., 112(2):187-213, 1993.

[19] G. Higman. Subgroups of finitely presented groups. Proc. Roy. Soc. Ser. A, 262:455475, 1961. 
[20] D. F. Holt, S. Rees, C. E. Röver, and R. M. Thomas. Groups with context-free co-word problem. J. London Math. Soc. (2), 71(3):643-657, 2005.

[21] J. E. Hopcroft and J. D. Ullman. Formal Languages and their Relation to Automata. Addison-Wesley, 1969.

[22] M. Kambites. Formal languages and groups as memory. arXiv:math.RA/0601061, 2006.

[23] M. Kambites. Word problems recognisable by deterministic blind monoid automata. Theoret. Comput. Sci., 362(1):232-237, 2006.

[24] M. Kambites, P. V. Silva, and B. Steinberg. On the rational subset problem for groups. J. Algebra (to appear), 2006.

[25] V. Mitrana and R. Stiebe. The accepting power of finite automata over groups. In New trends in formal languages, volume 1218 of Lecture Notes in Comput. Sci., pages 39-48. Springer, Berlin, 1997.

[26] D. E. Muller and P. E. Schupp. Groups, the theory of ends, and context-free languages. J. Comput. System Sci., 26(3):295-310, 1983.

[27] R. J. Parikh. On context-free languages. J. Assoc. Comput. Mach., 13:570-581, 1966.

[28] D. J. S. Robinson. A Course in the Theory of Groups, volume 80 of Graduate Texts in Mathematics. Springer-Verlag, second edition, 1996.

[29] D. Segal. Polycyclic Groups, volume 82 of Cambridge tracts in mathematics. Cambridge University Press, New York, 1983.

[30] J. R. Stallings. On torsion-free groups with infinitely many ends. Ann. of Math. (2), 88:312-334, 1968.

[31] A. J. Wilkie and L. van den Dries. An effective bound for groups of linear growth. Arch. Math. (Basel), 42(5):391-396, 1984. 